\title{
APPLYING THE AHP IN HEALTH ECONOMIC EVALUATIONS OF NEW TECHNOLOGY
}

\author{
Marjan Hummel*, Lotte Steuten, Karin Groothuis-Oudshoorn, Maarten IJzerman \\ Department of Health Technology \& Services Research \\ Univers ity of Twente, Enschede, The Netherlands \\ E-mail: j.m.hummel@utwente.nl \\ l.m.g.steuten@utwente.nl \\ c.g.m.oudshoorn@utwente.nl \\ $\underline{\text { m.j.ijzerman@utwente.nl }}$
}

\begin{abstract}
Much research in health care is devoted to health economical modelling. Even though the Analytic Hierarchy Process (AHP) is increasingly being applied in health care, its value to health economical modelling is still unrecognized. We explored the value of using AHP-derived results in a health economic model. We applied the AHP to provide input for a health economic evaluation of a new technology to diagnose breast cancer. No clinical data were available about the sensitivity and specificity of this technology. By means of the AHP, an expert panel estimated the sensitivity and specificity to be used in this model. Moreover, additional criteria including patient comfort and risks could be added to the health economic model. On the basis of the methodology suggested, the AHP proved to be feasible to support a comprehensive health economical evaluation of new technology, where clinical evidence is not yet available, or incomplete.
\end{abstract}

Keywords: Analytic Hierarchy Process, methodological development, health economic model, diagnostic technology

\section{Introduction}

Health economical modelling dominates the focus of research conducted in health care. In these studies, the expected clinical gains and benefits of applying new technology in health care are calculated. The analytic hierarchy process (AHP) is increasingly being applied in health care. Yet in health economical modelling, the potential value of the AHP remains largely unexplored. We studied the feasibility of using the AHP to support health economic evaluations of new technology.

Health economic evaluations are increasingly advocated for use during the development stage of new medical technologies, i.e. from initial idea up to phase III-like trials anticipating market access and reimbursement (Pietzsch \& Paté-Cornell, 2008; Vallejo-Torres, et al, 2010). The rationa le behind early modelling is to inform internal investment decisions to select potential products or prototypes to take forward, and to avoid investments in new technologies that are less likely to become successful. Ultimately, this should lead to effective and affordable technology to become available to patients more rapidly.

Yet, decision-analysis in the stage of technology development is typically challenged by a lack of data. In such a context, formal methods can be used to elicit expert knowledge on the parameters of interest to populate our decision models. One such methods is Saaty's analytic hierarchy process (AHP), a validated

\footnotetext{
* Corresponding author
} 
technique for multi-criteria decision analysis (Saaty, 1994). Dolan, et al (1991), Hummel, et al (2000), and IJzerman \& Hummel (2010) showed that the AHP is valuable to support health care decision making. We explored the AHP to 1) support the estimation of model inputs for which no empirical data are available yet, and 2) offer a weighting procedure to combine multiple (additional) criteria in the estimation of the outcomes of applying the new technology.

As a first illustration of our approach, we modeled the expected cost-effectiveness of a new imaging technology to diagnose breast cancer. This new technique is photo acoustic mammography (PAM). PAM uses a combination of light and ultrasound to detect breast cancer. In our example we assumed PAM to be a second line diagnostic, which is performed after the initial ultra-sound test was found to be positive. The AHP supported elicitation of expert know ledge to estimate the sensitivity, specific ity, patient comfort and risks of this new second line imaging technology to diagnose individuals suspected to have a breast tumor. These AHP-derived estimations for PAM were elicited relative to the current practice of using MRI as a second line diagnostic, and were used as inputs in a simple health economic model.

\section{Methods}

\subsection{The Analytic Hierarchy Process (AHP)}

The AHP supported elicitation of knowledge of an expert panel consisting of radiologists, oncologists, physicists and a nurse practitioner.

These experts first judged the importance of criteria related to the sensitivity, specificity, patient comfort and safety in assessing breast cancer imaging techniques. Regarding the sensitivity and specificity, the experts estimated how important multiple tissue and other physiological characteristics were in determining the sensitivity and specificity.

These characteristics included for example how well the technique could identify the shape of the tumor or the vascularization around the tumor. Furthermore, the experts compared the expected performance of PAM with the gold standard MRI on each of the identified characteristics.

The weighted average performance of the imaging techniques represented the expected relative sensitivity and specificity of PAM in comparison with MRI. Likewise, the new imaging technique PAM was compared to the performance of MRI concerning patient comfort and safety. Criteria related to patient comfort were body contact, environmental impact, and time between scan and result. Criteria related to safety are physical exposure, chemical exposure and bodily burden.

Figure 1 shows the criteria used to compare the performance of PAM with MRI. 


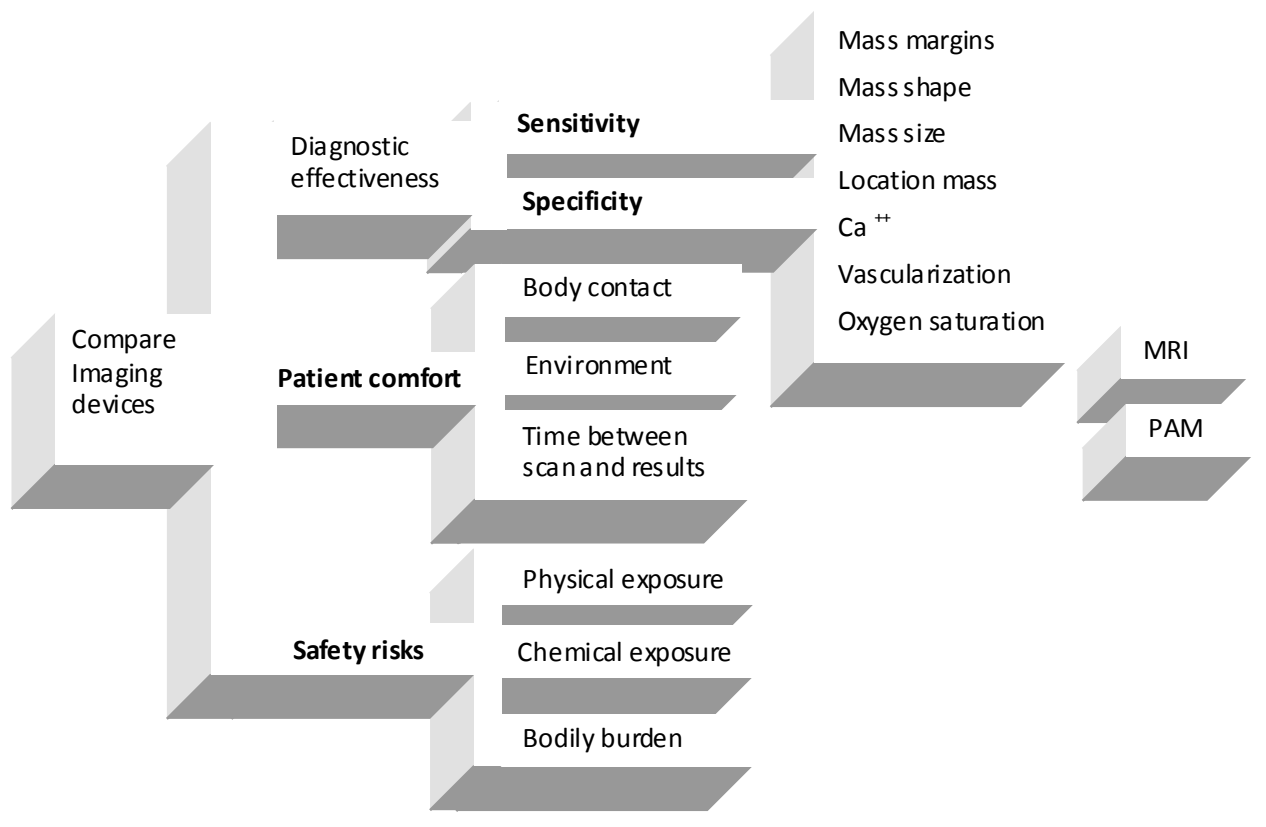

Figure 1: AHP structure to asses PAM in comparison with MRI

\subsection{The health economic decision model}

Markov decision models are commonly used to calculate the expected health economic outcomes of applying health care technology. The Markov decision model used in this illustration was adapted from a previously published Markov model (Retèl, et al, 2010), comparing the cost-effectiveness of the two diagnostic tests including MRI in a population of high risk patients, comparable to the population for which we derived the AHP-data. The structure of the model is shown in figure 2.

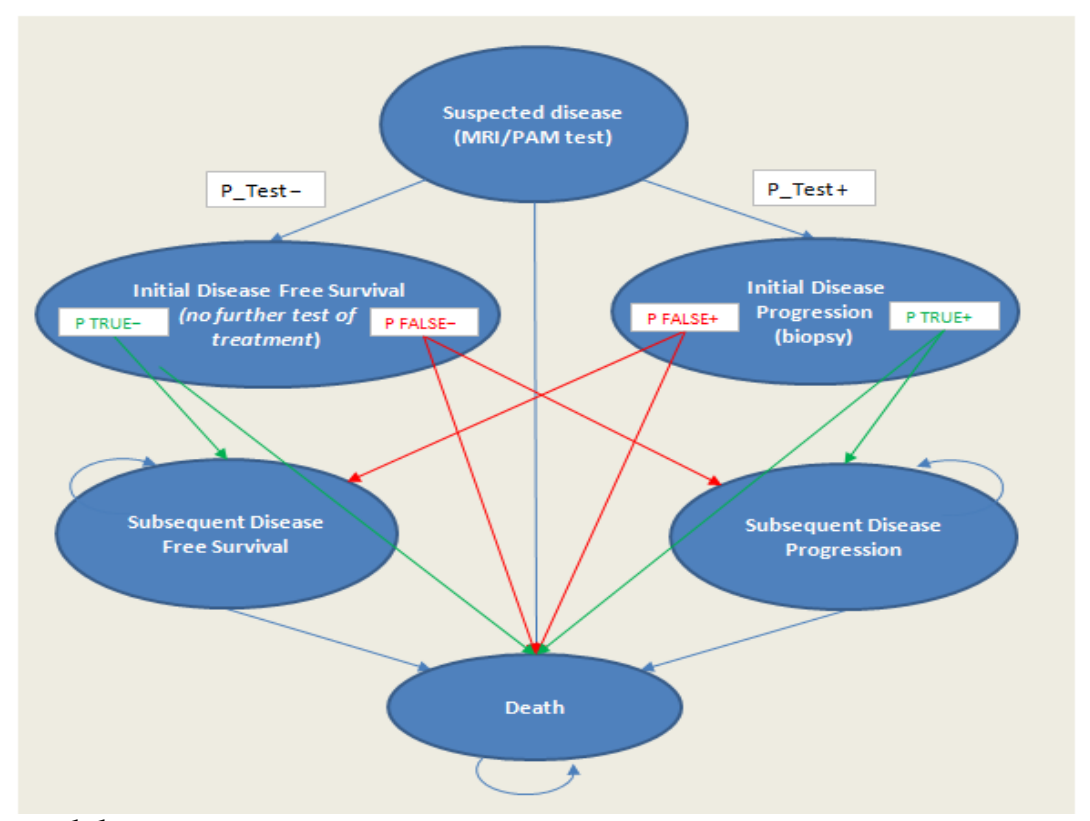

Figure 2. Markov model structure

In this health economic model, patients suspected to have breast cancer are diagnosed with either PAM or MRI. Those patients with a true positive diagnos is test outcome continue with the ir disease free survival. 
The patients with a false negative test outcome receive no biopsy and the ir disease progresses. Patients with a false positive test have a biopsy and continue with the ir disease free survival. Patients with a true positive diagnosis are treated and progress in their disease. The sensitivity and specificity of the diagnostic technology determine the amount of true negative, true positive, false positive and true positive test outcomes. Applying either PAM or MRI will consequently impact the probabilities for patients to be in one of the health states, and consequently will impact the quality of their life. Outcome measures are the quality adjusted life years gained of the patients and the costs involved.

\subsection{Methodol ogical challenges}

In using the AHP weights and priorities as inputs in the health economic model, we face two methodological challenges:

a) Converting the relative AHP-derived priorities for the sensitivity and specificity of PAM to absolute estimations of the sensitivity and specificity.

Sensitivity and specificity are used as direct inputs in the health economic model. By using an approximation of the priority value function of the AHP (figure 3), we calibrated the relative priorities for the sensitivity and specificity into absolute values for the sensitivity and specificity of PAM. We combined the relative priorities of PAM with the known absolute sensitivity and specificity of MRI (sensitivity $\mathrm{PAM}=0.864$; sensitivity $\mathrm{MRI}=0.860$; specif ic ity $\mathrm{PAM}=0.88$; specificity $\mathrm{MRI}=0.90$ ).

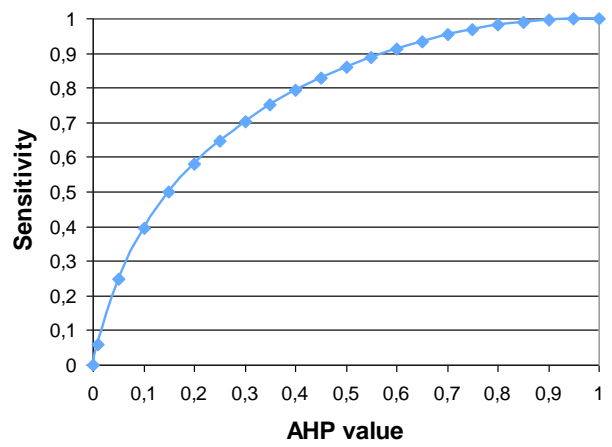

Figure 3. Conversion from relative sensitivity to absolute sensitivity

These estimations of the sensitivity and specificity of PAM were used as direct inputs in the Markov decision model.

b) Weighting the additional criteria patient and safety in the outcomes of the health economical model.

Since patient comfort and safety were no explic it parameters in the Markov model, we used the AHP derived estimations of patient comfort and safety as indirect inputs in the Markov decision model. By knowing the impact of sensitivity on health utility from the Markov model, and assuming that a criterion with a lower weight will have a lower impact on health utility, we estimated the impact of patient comfort and safety on health utility.

For: $\quad \Delta \mathbf{p}_{\text {(innovation - current practice) } \mathbf{n}}=\Delta \mathbf{p}_{\text {(innovation - current practice) sensitivity }}$

then: $\quad \Delta$ utility $_{\text {(innovation - current practice) } \mathbf{n}}=\mathbf{w}_{\mathbf{n}} / \mathbf{w}_{\text {sensitivity }} * \Delta$ utility $_{\text {(innovation - current practice) sensitivity }}$

Patient comfort can cause a temporary disutility in the period of the diagnostic test. Risks associated with the diagnostic test can cause temporary disutilities (for example allergic reaction) as well as long term disutilities (for example cell damage). The assumption that the weight of the criterion is positively 
correlated to the impact on health utility was validated by one-way sensitivity analyses of sensitivity and specificity on generated utility using the Markov model.

\section{Results}

\subsection{AHP-deri ved estimations}

The expert panel estimated the sensitivity of breast cancer imaging techniques to be most important (weight $=0.55$ ), followed by safety (weight $=0.26$ ), specificity (weight $=0.14$ ) and patient comfort (weight $=0.05$ ). The new technique is expected to have a minimally higher sensitivity than MRI has (priority PAM $=0.51$; priority MRI $=0.49$ ). Whereas PAM could be strong in detecting oxygen saturation, MRI is expected to detect the margins of the tumor mass better. The specificity of PAM is expected to be slightly lower than MRI (priority PAM $=0.46$; priority MRI $=0.54$ ). PAM is anticipated to be less uncomfortable to patients (priority PAM $=0.61$; priority $\mathrm{MRI}=0.39$ ), and safer than MRI (priority PAM $=0.77$; priority MRI $=0.33$ ). The MRI is expected to cause more chemical risks for the patient due to the contrast fluid used. Regardless of its diagnostic performance, PAM offers additional advantages compared to MRI regarding these two criteria of patient comfort and safety risks (figure 4).

Figure 4. AHP outcomes

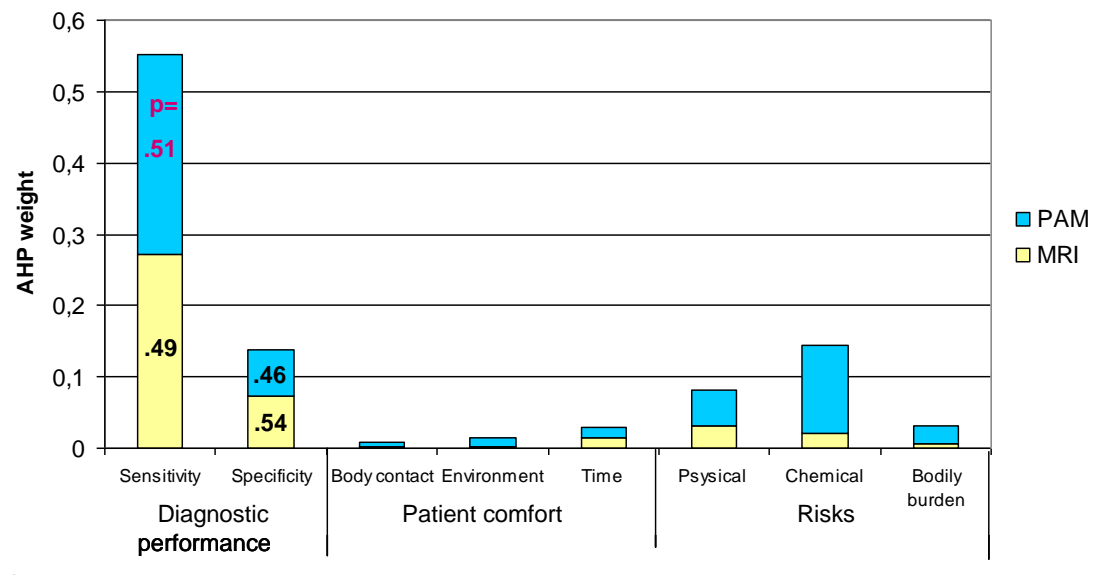




\subsection{Health economic outcomes}

Results of the base-case analysis (figure 5) show that there is hardly any difference between PAM and MRI in terms of quality adjusted life years per month in a simulated cohort of 1000 patients. The initial difference in the first two cycles is due to the higher sensitivity of PAM (i.e. more patients assumed to have breast cancer and receive confirmatory biopsy than in MRI) and higher costs of the PAM test. Quality of life and costs converge as soon as all patients have moved to the 'correct' health state, after which they are assumed to receive guideline treatment.

Accordingly, the initial incremental analysis on quality of life showed that, without considering the potential impact of patient comfort and safety risk criteria of PAM versus MRI on expected utility, the two technologies are likely on par. When including the relative performance of PAM on patient comfort and safety risks in the model, PAM might have a small benefit over MRI (figure 5).

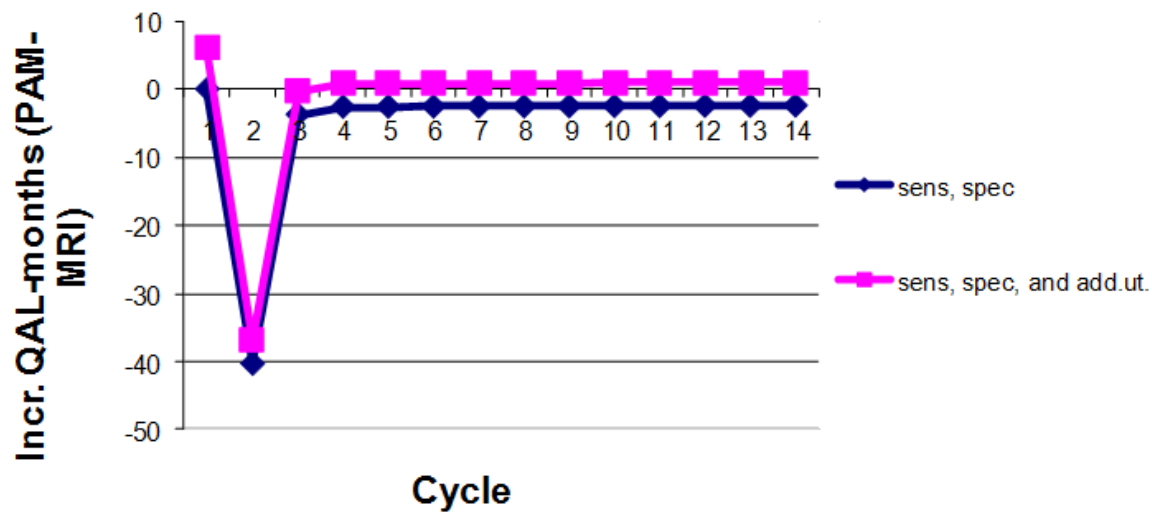

Figure 5. Health outcomes per month of PAM versus MRI

These findings imply that there is scope for PAM to be more effective than MRI, which at a certain price might lead to PAM being incrementally cost-effective. The finding may also suggest that in populations where criteria like patient comf ort and safety risks are relatively more important to future users than they are in the current "high suspection" population, e.g. in the context of population screening for breast cancer, PAM may more positively influence compliance to annual screening and hence improve incremental utility. Alternatively PAM may be positioned as the preferred diagnostic before MRI and subsequent biopsy, due to its higher sensitivity.

\section{Conclusions and discussion}

The use of the AHP in health economical modeling proved to be feasible. Based on the methodology suggested, the AHP can support the systematic assessment of relatively new technology, where clinical evidence is not yet available, or incomplete. Accordingly, the expected impact of the new breast cancer imaging technique on health outcomes could be estimated.

The AHP is appropriate to structure complex decisions. It decomposes the decision about the relative performance of a new technology compared to the gold standard in a series of pairwise comparisons. By comparing the performance regarding each determinant of sensitivity and specificity, a systematic approach is offered to estimate such complex performance parameters of diagnostic devices. In addition, the AHP allows the integration of additional effects of the imaging techniques in the decision model, besides the diagnostic performance. These effects can cause additional, often temporary disutilities to the health states of patients. In this specific case, additional disutilities caused by patients comfort and safety only had a low impact on health outcomes. In other cases however, one could image a stronger impact of factors such as patient discomfort. For example in the case of population screening, patient discomfort 
and risks associated with the health care intervention become relatively more important. Then the additional utility of PAM on these criteria may increase its future uptake in health care.

By improving elicitation of know ledge from experts in the Markov decision model, and adding additional criteria to the Markov decision model, we strive to enhance the predictive validity of early health economic models that need to be populated while having few to none empirical data. The AHP could be a valuable addition to the current methods for health economic evaluations of new technology.

\section{REFERENCES}

Pietzsch, J.B., Paté-Cornell, M.E. (2008). Early technology assessment of new medical devices. Int J Technol Assess Healthcare, 2(1), 36-44.

Vallejo-Torres, L., Steuten, L.M.G, Parkinson, B., et al. (2010). Integrating health economics into the product development cycle: a case study of absorbable pins for treating hallux valgus. Medical Decision Making, epub ahead of print.

Saaty, T.L. (1994). Highlights and critical points in the theory and application of the Analytic Hierarchy Process. European Journal of Operational Research, 74, 426-447.

Dolan, J.G, \& Bordley, D.R. (1991). Individualized Patient Decision-Making Using the Analytic Hierarchy Process (AHP) - Reliability, Validity, and Clinical Usefulness. Medical Decision Making, 11(4), 322.

Hummel, J.M., Rossum, W. van, Verkerke, GJ., et al. (2000). Assessing medical technologies in development. A new paradigm of medical technology assessment. Int J Technol Assess Health Care, 16(4), 1214-1219.

IJzerman, M.J., \& Hummel, J.M. (2010). Value of multi-criteria decision analys is in early assessment of diagnostic devices. Value in Health, 13(7), A429.

Retèl, V.P., Joore, M.A., Knauer, M., et al. (2010). Cost-effectiveness of the 70-gene signature versus St. Gallen guidelines and Adjuvant Online for early breast cancer. Eur J Cancer, 46, 1382-1391. 